\title{
Subject Index Vol. 13, 1997
}

Adrenalectomy 4333

Adrenalectomy, minimally invasive 4333

Anal carcinoma 145

Angiography S2 12

-, cruro-pedal arteries: postoperative,

preoperative, run-off S2 12 Antireflux surgery S2 106 -, experience, outcome 2138

Barostate 292 Bile duct lesions 156

strictures, postoperative: causes, the

rapy 156

- $\quad$ surgery, postoperative strategies 2158

Cancer incidence 3206 -, colorectal 122 Carcinoid 4274

- $\quad$ tumors 4 268,4 292

-, rectum, surgical therapy, symptoms 4268

Carcinoma, colorectal 3206

Chemotherapy, arterial 122

-, adjuvant, regional, systemic SI 50

Cholangiography, intraoperative 3228

Cholangioscopy 3222

Cholecystectomy, laparoscopic 3228

Choledocholithiasis 3228

Chronic arterial occlusive disease, stage III/ IV: conservative therapy, defibrinating agents, physical training, prostaglandin El, rheologica platelet aggregation inhibitors, vasodilatators S2 1

pancreatitis SI 12 Colon 4292

carcinoma 1 37, 3212

-, diagnosis, prognosis, recurrence, therapy 3212 Colorectal adenoma: follow-up 3206

Common bile duct injury 3228 Computed tomography (CT) 3176 Crural bypass surgery S2 31

reconstruction S2 15

vessels S2 19

CT during arterial portography (CTAP) 3176

Diabetes mellitus 3202

Drainage, transhepatic/transpapillary SI 43

Ductal pancreatic cancer SI 33 Dysphagia 2132

ECL cell 4260 
EHL 3222

Embolectomy, femoral, pedal, truncal S2 27

Embolization 4292

Endocrine pancreas tumors: computed tomography (helical technology) magnetic resonance imaging (fast imaging) 4301

Endoscopic retrograde cholangiopancrea-tography SI 6

Endosonography SI 6

Esophagealcancer 16

-, therapy, additiv, adjuvant, neoadju-vant 16

Esophageal motility 2132

Esophageal sphincter, lower 286

ESWL 3222

Femoro-crural bypasses: cumulative patency rate, early occlusion, immediate occlusion of bypass S2 6

Fluorodeoxyglucose SI 10

Fundoplication, laparoscopic 2106

-, - partial 2117

Gastinoma: diagnostics, surgical therapy 4313

Gastric cancer: advanced tumor stages, surgery, treatment modalities (adjuvant, preoperative)

114

carcinoid 4260

motility 292

secretion 292 Gastrin 4260 Gastrinoma SI 36 Gastroesophageal reflux disease (GERD)

2106,2117,2132,2138, 2143

, cost benefit analysis, drug therapy,

fundoplication, surgery 2147

, current drug therapy, laparoscopic

fundoplication, minimally invasive surgery 2143

, surgical therapy 2110

Gastrointestinal neuroendocrine tumors: alpha-interferon, chemoembolization,

chemotherapy, hormone secretion, oc-treotide, somatostatin receptors 4308

Gene mutations SI 16

GERD 2 132, 2138

Growth factor receptors SII 16

- factors SI 16

Helicobacter pylori 292 Hepatic arteries 4292 Hepaticolithiasis 3222 Hepatocellular

carcinoma 3176 Hernioplasty, laparoscopic: complications,

recurrences 167 Histamine 4260 Hormones 286

Insulinoma 4 318, SI 36 -, surgical therapy 4318 Intrahepatic stones 2158 ITA S2 19

Laparoscopic fundoplication, technique 2124

Laparoscopy 2117

Laserangioplasty S2 19

Liver: carcinoid tumor, embolization 4286

-, hemangioma, tumor, cyst 3176

-, metastases 122, 3 176,4 286

-, secondary neoplasms, surgery, transplantation 4279 
-, transcatheter arterial embolization 4286

Longterm survival 3202

Lymph node and connective tissue dissection SI 33

Magnetic resonance imaging (MRI) 3176

Malignancies, gastrointestinal: computed tomography, staging 3186

Malignant carcinoid syndrome 4279

Management 4260

Melanoma, malignant: indication for surgical treatment, viscera! metastases 3218

MEN I 4260

Metal stents SI 43

Motility, antroduodenal 286

Nissen fundoplication: long-term results, side effects 2110

KAflGER

(C) 1997 S. Karger GmbH, Freiburg Fax (0761) 4520714 http://www.karger.ch

34')

Occlusive jaundice SI 43 Organ preservation 145

Palliative surgery SI 24

Pancreas and kidney transplantation 3202

- $\quad$ carcinoma SI 10, SI 46

-, radiochemotherapy, radiotherapy SI 46

- $\quad$ tumors, endocrine SI 36

Pancreatectomy, regional, total SI 24

Pancreatic cancer SI 6, SI 12, SI 16,

SI 24, SI 43, SI 50

- $\quad$ carcinoma, exocrine: grading, R classifi

cation, TNM classification, typing SI 1

- $\quad$ transplantation 3202

Pancreatitis SI 10

Pancreatoduodenectomy (Whipple's resection) SI 24

Peritonitis 151 Peroneal artery S2 15

Polypectomy 3206

Positron emission tomography (PET) SI 10 Precancerosis SI 12 PTA S2 19 PTCD 3222

PTFE bypass S2 31

Pylorus-preserving partial pancreatoduodenectomy SI 24

Radiochemotherapy 145

Reconstruction of arteries: complications (intraoperative postoperative), infection S2 40

Rectal carcinoma 137

Reflux, gastroesophageal, duodenogastric 286

- $\quad$ disease 2 132, 298 , diagnostic, omeprazole test, $\mathrm{pH}-\mathrm{ma}-$

nometry, symptoms 298

- $\quad$ esophagitis 292

Scoring systems: dAPS, TISS 151

Small intestine 4292

Smoking SI 12 
Somatostatin receptor scintigraphy 4274

Surgery, minimal invasive: complications,

intraabdominal infection, peritonitis

162

Therapy, adjuvant, neoadjuvant 122,137 -, neoadjuvant 137 Toupet fundoplication 2132 Ultrasound 3176

Vascular surgery S215 Venous bypass S2 31

350

Chir Gastroenterol 1997;13

Subject Index Vol. 13, 1997 